\title{
Ferulic Acid Induces Bone Marrow Mesenchymal Stem Cells to Alleviate the Activation of Hepatic Stellate Cells and Liver Fibrosis via Cytoskeletal Rearrangement Inhibition and Mir-19b-3p Transfer
}

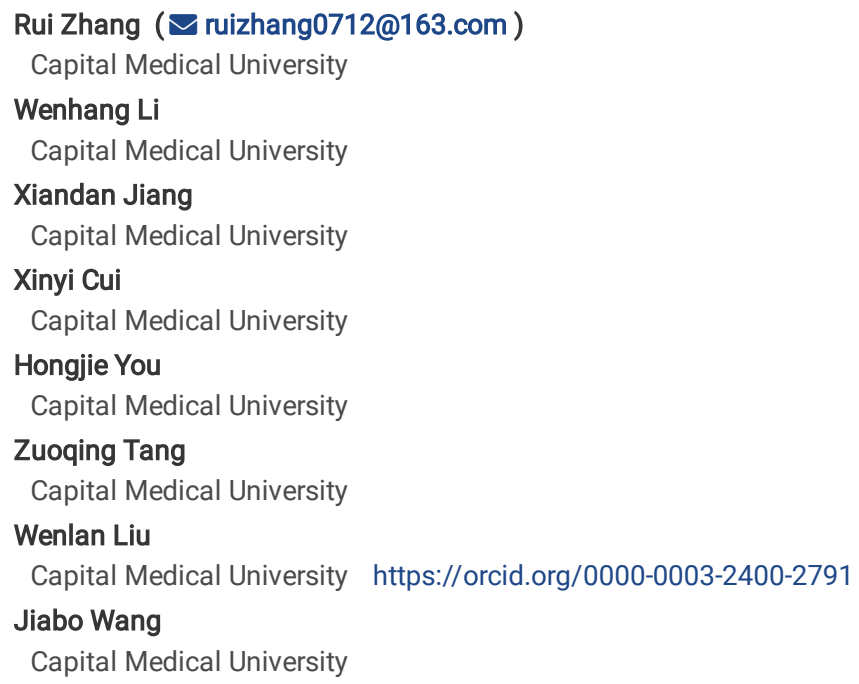




\section{Abstract}

Background: Bone marrow mesenchymal stem cells (BMSCs) are effective for treating fibrotic liver. BMSCs contain a variety of proteins and RNAs, which have functions similar to their derived cells, but the specific mechanism is unclear. In a recent study, ferulic acid (FA) was highly effective in treating liver fibrosis. Therefore, we combined BMSCs and FA to treat $\mathrm{CCl}_{4}$-induced fibrosis models.

Methods: First, we used BMSCs and FA to treat $\mathrm{CCl}_{4}$-induced fibrosis models and observed their therapeutic effect, investigated the specific mechanism of this combination therapy in liver fibrosis. Second, we created a BMSC/hepatic stellate cell (HSC) co-culture system and used FA to treat activated HSCs. We next used cytochalasin D and angiotensin II to investigate whether BMSCs and FA inactivate HSCs through cytoskeletal rearrangement. MiR-19b-3p was enriched in BMSCs and targeted TGF- $\beta$ receptor II (TGF- $\beta$ R2). We transfected miR-19b-3p into HSCs and BMSCs separately and detected whether BMSCs transferred miR-19b-3p to HSCs or inactivated HSCs.

Results: We used BMSCs and FA to treat $\mathrm{CCl}_{4}$-induced fibrosis models and found that the combination therapy had better effects than $\mathrm{FA}$ or $\mathrm{BMSC}$ alone. The expression of the profibrotic markers a-SMA and COL1-A1 was significantly decreased in HSCs co-cultured with BMSCs and FA treatment. Cytoskeletal rearrangement in HSCs was inhibited, and RhoA/ROCK pathway gene expression was decreased. With angiotensin II treatment, COL1-A1 and a-SMA expression increased, while with cytochalasin D treatment, profibrotic gene expression decreased in HSCs. COL1-A1, a-SMA and RhoA/ROCK pathway genes were decreased in activated HSCs treated with a miR-19b-3p mimic, indicating that miR-19b-3p inactivated HSCs by suppressing RhoA/ROCK signalling. In contrast, profibrotic genes were significantly decreased in BMSCs treated with the miR-19b-3p mimic or a miR-19b-3p inhibitor and FA compared with BMSCs treated with the miR-19b-3p mimic alone.

Conclusion: BMSCs attenuated HSC activation and liver fibrosis by inhibiting cytoskeletal rearrangement and delivering miR-19b-3p to activated HSCs, inactivating RhoA/ROCK signaling. FA-based combination therapy showed better inhibitory effects on HSC activation, suggesting that BMSCs and their miRNAs combined with FA are novel antifibrotic therapeutics for treating chronic liver disease.

\section{Introduction}

Although the aetiologies of liver diseases are varied, a common pathological feature of most chronic liver diseases is liver fibrosis, which is characterized by the progressive replacement of functional hepatic tissue with extracellular matrix $(E C M)^{[1,2]}$. Hepatic stellate cells (HSCs) are a key fibrogenic cell type that contributes to liver fibrosis. Upon liver injury, Kupffer cells and endothelial cells in the liver release cytokines, such as transforming growth factor- $\beta$ (TGF- $\beta$ ), to activate quiescent HSCs. Activated HSCs transdifferentiate into myofibroblastic HSCs ${ }^{[3]}$. Then, the cytoskeleton in these HSCs undergoes rearrangement, the cell dynamics increase, and these cells migrate to the site of liver injury and produce substantial amounts of ECM components, such as a-smooth actin (aSMA) and collagens. Fired-Man proved that activated HSCs are the main source of extracellular collagen in the liver. Hence, regulating HSC activation and migration could be a potential antifibrotic therapeutic strategy.

Bone marrow mesenchymal stem cells (BMSCs) have become an emerging therapeutic agent for liver fibrosis in recent years due to their advantages of proliferative potential, low immunogenicity, abundant sources, and tissue repair, chemotactic and homing abilities ${ }^{[4]}$. Many studies have reported that the therapeutic effects of mesenchymal stem cells (MSCs) against liver fibrosis/cirrhosis are related to the capacity of these cells to undergo hepatocyte-like differentiation, exert immunomodulatory activities, and secrete paracrine factors. Infusion of $\mathrm{BMSCs}$ was reported to ameliorate $\mathrm{CCl}_{4}$-induced liver damage and fibrosis in rats via the FGF2-DIk1-Notch1 pathway ${ }^{[5]}$. Due to the cytoskeletal changes that occur when HSCs are activated, we hypothesized that BMSCs could ameliorate liver fibrosis by inhibiting cytoskeletal rearrangement. In addition, many recent studies have proven that BMSCs can produce microRNAs $(\text { miRNAs })^{[6]}$. Feng et al. demonstrated that MSC-derived miRNAs reduced infarct size and cardiac fibrosis by inhibiting apoptosis in mice with myocardial infarction. miR-125b contained in exosomes derived from chorionic plate-derived MSCs (CP-MSCs) ameliorated hepatic fibrosis by suppressing hedgehog $(\mathrm{Hh})$ signalling, which is an essential regulator in liver fibrosis ${ }^{[7,8]}$. These findings suggest that miRNAs play important roles in MSC-mediated tissue repair and regeneration.

Ferulic acid (FA), a derivative of cinnamic acid, has therapeutic activity against a variety of diseases. The antioxidant effects of FA have also been

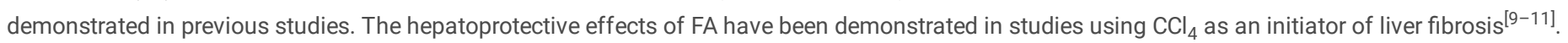

The protective mechanisms of BMSCs in liver fibrosis have been studied, but few reports have studied the inhibition of cytoskeletal rearrangement in liver fibrosis. Of the studies performed, single BMSC treatment for liver diseases has shown inconsistent efficacy. Therefore, the mechanisms underlying the antifibrotic effects of BMSCs in combination with FA were investigated in the current study.

\section{Materials And Methods}

\section{Cell culture}

SD-BMSCs (purchased from FineTest, Wuhan, China) were cultured in Dulbecco's modified Eagle's medium (DMEM; Gibco, Thermo Fisher Scientific, MA, USA) supplemented with $10 \%$ fetal bovine serum (FBS; AQ, China) and $1 \%$ penicillin/streptomycin (P/S; Gibco) at $37^{\circ} \mathrm{C}$ in a humidified atmosphere containing $5 \%$ $\mathrm{CO}_{2}$. HSC-T6 cells (purchased from KeyGEN) were cultured in DMEM-high (Gibco) supplemented with $10 \% \mathrm{FBS}(\mathrm{AQ})$ and $1 \% \mathrm{P} / \mathrm{S}(\mathrm{Gibco})$ at $37^{\circ} \mathrm{C}$ in a humidified atmosphere containing $5 \% \mathrm{CO}_{2}$. SD-BMSCs and HSC-T6 cells (purchased from Keygen Biotech, Nanjing, China) were seeded in 25-cm² plates at $70 \%$ confluence. 


\section{Cell co-culture experiments}

HSCs were activated with TGF- $\beta 1$ (10 ng/mL, PeproTech, Germany) for $24 \mathrm{~h}$. HSC and BMSC co-culturing was conducted using transwell inserts (Corning, NY, USA), which were permeable to the culture medium but not the cells. All co-culture experiments were performed with HSCs seeded in the bottom chambers and BMSCs seeded in the top chambers. The insert chambers containing BMSCs were transferred into a co-culture system and cultured for $12 \mathrm{~h}$. The cells were divided into 6 groups (NC, Model, Positive, FA-1 mg, FA-0.5 mg, and FA-0.25 mg). Except for those in the NC group, HSCs were treated with different doses of FA $(1 \mathrm{mg} / \mathrm{mL}, 0.5 \mathrm{mg} / \mathrm{mL}$, or $0.25 \mathrm{mg} / \mathrm{mL})$ and colchicine $(6 \mu \mathrm{g} / \mathrm{mL})$ for $24 \mathrm{~h}$.

\section{Cytoskeletal inhibitor and agonist treatment experiments}

HSCs and BMSCs were co-cultured with transwell inserts for $12 \mathrm{~h}$. Then, the HSCs, except for those in the NC group, were activated with TGF- $\beta 1$ (10 ng/mL) for $24 \mathrm{~h}$. After activation, the cells, except those in the NC and model groups, were treated with $1 \mu \mathrm{M}$ cytochalasin D (cytoskeleton inhibitor, purchased from Sigma, Germany) or $10 \mu \mathrm{M}$ angiotensin II (cytoskeleton agonist, purchased from Sigma, Germany) for $24 \mathrm{~h}$.

\section{Transfection of miR-19b-3p mimics and a miR-19b-3p inhibitor into HSCs and BMSCs}

BMSCs and HSCs were transfected with $20 \mu \mathrm{g} / \mathrm{mL}$ rat miR-19b-3p mimics (5'-UGU GCA AAU CCA UGC AAA ACU GA-3'; KeyGEN), miR-19b-3p inhibitor (5'-UCA GUU UUG CAU GGA UUU GCA CA-3'; KeyGEN), miR-mimics NC (KeyGEN) or miR-inhibitor NC (KeyGEN) using an electronic transient transfection machine (BTX Genimi X2, USA). HSCs were divided into 6 groups: normal, model, HSC+mimics (mimic), HSC+inhibitors (inhibitor), HSC+ mimics NC (NC-d), and HSC+ inhibitor NC (NC-s); while BMSCs were divided into 8 groups: normal, model, BMSC+mimics (mimic), BMSC+inhibitors (inhibitor), BMSC+mimics +FA (mimic+FA), BMSC+inhibitors+FA (inhibitor+FA), BMSC+ mimics NC (NC-d), and BMSC+ inhibitor NC (NC-s). Cells were observed and captured using an inverted fuorescence microscope (Axio observer A1, Zeiss, Germany).

\section{Elisa Assay}

We extracted the culture medium and centrifuged (10000 $\mathrm{g}, 20 \mathrm{~min})$ to remove the cell debris. Then we detected the content of COL1-A1 and a-SMA in culture medium with enzyme linked immunosorbent assay kits (mlbio, China) according to the manufacturer's protocol. All data are presented as the mean \pm SEM of values from at least three repetitive experiments.

\section{Cloning of vector constructs and luciferase reporter assay}

For cloning of vector constructs, target genes of Rat miR-19b-3p were predicted by bioinformatics analysis using the online database: http://mirwalk.umm.uniheidelberg.de. The 3' UTR of rat TGF- $\beta$ R2 from genomic DNA, containing binding sites for rat miR-19b-3p, was amplified by PCR. The primers sequences used for vector construction were as follows: forward,

5'TTCTAGTTGTTTAAACGAGCTCGCTAGCCTCGAGCTTTTTCTGGGCAGGCTGGGCCAAGACTCCG3',reverse,5'GCAGCCGGATCAGCTTGCATGCCTGCAGGTCGACA $3^{\prime}$. The PCR product was purified using an AccuPrep PCR purification kit (Tiangen), cut by the restriction enzymes Xho1 and Sall, and then cloned into the pmirGLO vector (Promega, WI, USA). The vector constructs with 3' UTR of TGF- - $\beta$ R2 were transformed into Escherichia coli, and then plasmid DNA was extracted from well-transformed, ampicillin-resistant E. coli, using an AccuPrep plasmid mini extraction kit (Tiangen, China). The sequences of the miR-19b-3pbinding sites of the 3'UTR of TGF- $\beta R 2$ were confirmed by sequencing analysis (KeyGEN, China). Mutant vectors lacking the miR-19b-3p-binding site were manufactured by KeyGEN (KeyGEN, China). For the luciferase reporter assay, HSC-T6 cells were seeded in 24-well culture plates in culture medium without P/S 1 day before transfection. Using an electronic transient transfection machine (BTX Genimi X2, USA), cells were transfected with a mixture of pmirGLO vector construct and either 20 ug miR-19b-3p mimic (KeyGEN) or scrambled miRNA (NC-d; KeyGEN) as an NC. At $48 \mathrm{~h}$ after transfection, cells were harvested and tested with the Dual-Luciferase reporter assay system (Beyotime, China) according to the manufacturer's protocol. All firefly luciferase activity data are normalized to Renilla luciferase activity and presented as the mean \pm SEM of values from at least three repetitive experiments.

\section{Experimental animal models}

Male Sprague-Dawley rats were treated as per the National Institute of Health Guidelines for the Care and Use of Laboratory Animals. All experiments were approved and supervised by the Animal Care and Use Committee of Capital Medical University (approval number: SCXK 2016-0006), housed with a 12-h light/12-h dark cycle and allowed free access to normal food and water. Eight-week-old rats received $0.2 \mathrm{~mL} / \mathrm{kg}$ of body weight CCl 4 (Jin Chemical Pharmaceutical, Seoul, Korea) dissolved in olive oil by intraperitoneal injection, were then injected with $0.1 \mathrm{~mL} / \mathrm{kg}$ of body weight $\mathrm{CCl}_{4}$ for 2 weeks and were finally injected with $0.05 \mathrm{~mL} / \mathrm{kg}$ of body weight $\mathrm{CCl}_{4}$ for 2 weeks. All injections were administered three times per week ( $\mathrm{n}=30$ ). As a control (NC group), mice were injected with an equal volume of olive oil $(\mathrm{n}=6)$. Next, we randomly chose $2 \mathrm{CCl}_{4}$ groups to inject with $5 \otimes 10^{6} \mathrm{BMSCs}$ (stained with CMSE, meilunbio, China). After that, we chose a $\mathrm{CCl}_{4}$ group and a $\mathrm{CCl}_{4}+\mathrm{BMSC}$ group to treat with $\mathrm{FA}(10 \mathrm{mg} / \mathrm{kg})$ and another $\mathrm{CCl}_{4}$ group to treat with colchicine $(0.1 \mathrm{mg} / \mathrm{kg})$ as a positive control. The other groups were treated with equal amounts of normal saline. After treatment for 2 weeks, all mice were sacrificed, and the serum and liver tissues were obtained. The groups were as follows: normal control group (NC), model group (model), positive control group (positive), ferulic acid group (FA), BMSC group (BMSCs), BMSC+ ferulic acid group (FC). 


\section{Measurement of aspartate aminotransferase and alanine aminotransferase}

We collected $1 \mathrm{~mL}$ of blood from each animal. After storage at room temperature for $4 \mathrm{~h}$, the serum was separated by centrifugation ( $1500 \times \mathrm{g}, 1 \mathrm{~min})$. Then, $200-\mu \mathrm{L}$ serum samples were taken for analysis. The activities of serum alanine aminotransferase (ALT) and aspartate aminotransferase (AST) were determined using commercial kits obtained from Konkahongyuan Co. (Beijing, China) according to the manufacturer's instructions.

\section{Histopathological evaluation}

Fresh liver tissue samples were fixed in $10 \%$ formaldehyde at $4^{\circ} \mathrm{C}$ for $12 \mathrm{~h}$ and embedded in paraffin wax for histological evaluation. Sections were stained with haematoxylin and eosin (HE), and the severity of histological changes was evaluated. We obtained images by using a microscope equipped with a LICOR Odyssey Infrared Imaging System (LI-COR, Inc., Lincoln, NE, USA).

\section{Masson trichrome staining}

For staining, 4 to 6 um-thick sections were cut from paraffin-embedded blocks and stained with Masson trichrome (MT) and FANCM. For Masson trichrome staining, the Trichrome III Green Staining Kit, a modified version of the Masson trichrome stain, was used with Bouin's Solution application to intensify the final coloration.

\section{BMSCs tracking in vitro}

Liver tissue (selected from BMSCs group) was soaked into $4 \%$ PFA in $4^{\circ} \mathrm{C}$ for $24 \mathrm{~h}$, then liver was soaked into $30 \%$ sucrose solution for $48 \mathrm{~h}$. After washed with PBS for 3 times, the tissue was frozen into liquid nitrogen and sliced into 4 um sections. Sections were observed and captured using confocal laser scanning microscope (Zeiss SP8-STEAD, Germany).

\section{Tissue Clearing}

Liver tissue (selected from BMSCs group) was soaked into $4 \%$ PFA in $4^{\circ} \mathrm{C}$ for $24 \mathrm{~h}$, then washed with PBS for $2 \mathrm{~h}$ at RT for 3 times. After tissue fixation, the tissue was soaked into 50\% Cubic-L (TCl, Tokyo Japan) for $24 \mathrm{~h}$, then soaked into Cubic-L for $48 \mathrm{~h}$ at $37^{\circ} \mathrm{C}$. After washed with PBS for 3 times, the tissue was soaked into 50\% Cubic-R+ (TCl, Tokyo, Japan) for $24 \mathrm{~h}$ and then soaked into Cubic-R+ for $48 \mathrm{~h}$. The tissue was observed with lightsheet microscopy (Abberior instruments, USA).

\section{Quantitative real-time PCR analysis}

We extracted total RNA from frozen liver tissues using a Trizol reagent kit (Tiangen Biotech, Beijing, China) and then reverse transcribed the isolated RNA into cDNA using a reverse transcription kit (Tiangen Biotech, Beijing, China). To assay target gene expression, we used quantitative polymerase chain reaction (qRT-PCR) with a SYBR Green Taq kit (KAPA Biotechnology) on a 7500HT fast real-time PCR system (ABI, Foster City, CA, USA). The primers were designed using Primer 5 (Table 1). The PCR conditions were as follows: (1) $95^{\circ} \mathrm{C}$ for $30 \mathrm{~s}$ and (2) 40 cycles of $95^{\circ} \mathrm{C} \mathrm{for} 20 \mathrm{~s}, 60^{\circ} \mathrm{C}$ for $20 \mathrm{~s}$, and $72^{\circ} \mathrm{C}$ for $20 \mathrm{~s}$. The experiment was repeated three times.

Table 1

Primer sequences 


\begin{tabular}{|ll|}
\hline Primer name & Sequences 5'-3' $^{\prime}$ \\
\hline GAPDH-F & CTGGAGAAACCTGCCAAGTATG \\
\hline GAPDH-R & GGTGGAAGAATGGGAGTTGCT \\
\hline ROCK1-F & CAAAAATGATCAGTGGGCTTGG \\
\hline RoCK1-R & CCTACAAAAGGTAGCTGATTGCC \\
\hline LIMK1-R & CACAGTCACCCTCGTGTCTATCC \\
\hline RhoA-F & TCTCGTCCAGCGGCACATT \\
\hline RhoA-R & GATGGAGCTTGTGGTAAGACATGC \\
\hline SRF-F & GGCTGTCGATGGAAAAACACATC \\
\hline SRF-R & GCGGCGTTACACGACCTTC \\
\hline COL1-A1-F & TCTGAATCAGCGCCTTGCC \\
\hline COL1-A1-R & CACCTACAGCACGCTTGTGG \\
\hline a-SMA-F & GATTGGGATGGAGGGAGTTTAC \\
\hline a-SMA-R & ACCCAGGCATTGCTGACAG \\
\hline U6-F & AGAAGCATTTGCGGTGGAC \\
\hline U6-R & CTCGCTTCGGCAGCACA \\
\hline miR-19b-F & AACGCTTCACGAATTTGCGT \\
\hline miR-19b-R & CTCAACTGGTGTCGTGGAGTCGGCAATTCAGTTGAG TCAGTTTT \\
\hline Universal primer-A & ACACTCCAGCTGGGTGTGCAAATCCATGCAA \\
\hline
\end{tabular}

\section{Cytoskeleton staining}

HSCs were treated with TGF $\beta 1$ and then co-cultured with BMSCs. After the co-culture period, the cells were washed twice with PBS, fixed with $4 \%$ paraformaldehyde and permeabilized with $0.1 \%$ Triton X-100 in PBS for 20 min at room temperature. Then, the cells were stained with phalloidin (1:200) for 30 min. After phalloidin staining, the cell nuclei were stained with DAPI (1:100) for $10 \mathrm{~min}$. Cells were observed and captured using confocal laser scanning microscope (Zeiss SP8, Germany).

\section{Western blot analysis}

Cells and liver tissues were lysed in RIPA buffer (Lablead, Beijing, China), and total protein was extracted. The protein concentration was measured using a BCA protein assay kit (Beyotime, Nanjing, China). Protein probing by Western blot analysis was performed routinely; membranes were incubated with primary antibodies against a-SMA (1:1000; Sigma), Col1a1 (1:1000; Abcam, USA), GAPDH (1:1000; Cell Signaling Technology, USA), ROCK (1:1000; CST), RhoA (1:1000; CST), LIMK1 (1:1000, CST), SRF (1:1000, CST), and TGF- $\beta R 2$ (1:1000, CST) overnight at $4^{\circ} \mathrm{C}$. Then, an HRP-conjugated goat anti-rabbit lgG (1:10000; Jackson ImmunoResearch, USA) secondary antibody was incubated at $37^{\circ} \mathrm{C}$ for $1 \mathrm{~h}$. The immunobands were visualized using a chemiluminescent $\mathrm{HRP}$ substrate (NCM, Beijing, China). Finally, the immunoreactive protein bands were detected and imaged by Image Lab system (Bio-Rad ChemiDoc XRS, USA; Fusion FX, VILBER, France). Protein expression was standardized to that of GAPDH and quantified using Gel-Pro Analyzer software (Media Cybernetics, Rockville, MD, USA).

\section{Immunohistochemistry analysis}

All tissue samples were routinely fixed in $4 \%$ formalin and embedded in paraffin. The paraffin sections were dewaxed in xylol and rehydrated in a series of ethanol solutions (95\%, 80\%, and $70 \%$ ). Antigen retrieval was performed in a microwave oven with citrate buffer at pH 6.0 for 20 min and then cooled for 20 min. Endogenous peroxidases were blocked using solutions provided with the Reveal Kit (Biogen Cambridge, MA). After this procedure, we used $10 \%$ normal goat serum to block the sections for $1 \mathrm{~h}$ at $37^{\circ} \mathrm{C}$. The blocked sections were then incubated with the primary antibody overnight at $4{ }^{\circ} \mathrm{C}$. After incubation with primary antibodies against a-SMA (1:500), the sections were incubated with Reveal buffer. After 10 min, the sections were incubated with the secondary antibody for $1 \mathrm{~h}$. The reaction was stained with diaminobenzidine (DAB) and counterstained with hematoxylin. The sections were then hydrated in distilled water and dehydrated in a series of alcohol solutions and xylol. The slides were then covered with coverslips with the aid of Entellan (Merck, Germany). The experiment was repeated three times. 


\section{Statistical analysis}

Statistical analysis results are expressed as the mean \pm SEM. Statistical differences were analyzed by one-way and two-way ANOVA (SPSS 20.0, Chicago, IL, USA). $P$ values $<0.05$ were considered statistically significant.

\section{Results}

\section{FA promoted the ability of BMSCs to decrease serum AST and ALT levels in liver fibrosis models}

As shown in Figure 1a, we found that the liver was dark red, the liver surface was rough, and adhesions appeared between the liver lobes in the $\mathrm{CCl}_{4}$-treated group compared to the normal group. In contrast, with BMSC and FA treatment, the liver changed substantially. The color of the liver was returned to light red, the liver surface was smooth, and adhesions were reduced in the BMSC+FA group compared to the model group. Rat weight also showed differences among the groups. Compared to that of normal control rats, the weight of model rats was significantly decreased $(p<0.001)$. Weight was significantly increased with FA and BMSC treatment ( $<<0.001$, vs. the model group) (Figure 1b). The serum ALT and AST levels in model rats were significantly increased ( $<<0.05$, vs. control group), indicating successful establishment of the liver fibrosis rat model. FA, BMSC or FA\&BMSC administration significantly decreased serum AST and ALT levels ( $p<0.05$, vs. the model group) (Figure $1 c-d)$.

\section{Histopathological Assessment Of Liver Damage}

To further confirm the antifibrotic effects of BMSCs, FA and their combination in an in vivo rat liver fibrosis model, we performed HE and Masson staining. As shown in Figure 2a and 2b, inflammatory cell infiltration, fragmented hepatic nuclei, and collagenous fibre formation were observed in the model group. Damage caused by $\mathrm{CCl}_{4}$ was significantly attenuated by BMSCs, FA, and their combination. In addition, the combination treatment achieved better reductions in collagenous fibres compare to FA and BMSCs single group.

\section{Tracking Bmscs In Vivo}

BMSCs were pre-labelled with CMSE (green fluorescence, $492 \mathrm{~nm}$ ) before injection. To identify successful BMSC injection and BMSC localization, we extracted liver tissue for cryo-sectioning and tissue clearing. As shown in Figure 3a, liver sections contained clearly visible CMSE-labelled BMSCs throughout. As shown in Figure 3b, the CMSE-labelled BMSCs were mostly located around intrahepatic vessels.

\section{FA promotes the ability of BMSCs to improve liver fibrosis via the RhoA/ROCK pathway}

We identified that FA could promote the ability of BMSCs to reduce liver fibrosis via the RhoA/ROCK pathway. Compared with the normal group, the protein expression of the model group showed significant increases in the profibrotic markers a-SMA and COL1-A1, while in the FA and BMSC-treated group, their expression was decreased. In addition, FA combined with BMSCs showed better effects than the other two treatments. The expression patterns of RhoA and ROCK showed the same trends (Figure 4a-d).

QRT-PCR assays confirmed the protein data, showing reductions in a-SMA, COL1-A1, RhoA and ROCK expression in the FA, BMSCs and FA\&BMSC (FC) groups. These results clearly indicated that BMSCs combined with FA could be a novel treatment for liver fibrosis (Figure 4e).

\section{FA promotes the ability of BMSCs to inhibit cytoskeletal rearrangement in HSCs}

Whether FA promotes the ability of BMSCs to inhibit cytoskeletal rearrangement in HSCs was investigated. Co-cultured HSCs were evaluated by fluorescence and confocal microscopy and demonstrated successful uptake. Compared to those in the normal group, activated HSCs exhibited F-Actin remodelling to form a large number of thick stress fibres across the whole cell. In the FA-treated group, F-Actin was mainly distributed in peripheral cells, and a few fine stress fibres were found in these cells (Figure 5).

\section{FA promotes the ability of BMSCs to inhibit the activation of HSCs via the RhoA/ROCK/SRF and RhoA/ROCK/LIMK1 pathways}

To investigate the promotive effect of FA on the ability of BMSCs to inhibit the activation of HSCs, activated HSC-T6 cells cultured with an equal proportion of BMSCs were used as the model group, and activated HSC-T6 cells co-cultured with BMSCs and treated with different doses of FA ( $1 \mathrm{mg} / \mathrm{mL}, 0.5 \mathrm{mg} / \mathrm{mL}$, or $0.25 \mathrm{mg} / \mathrm{mL}$ FA) were used as the treatment groups. Inactivated HSCs co-cultured with BMSCs in normal culture medium were used for the normal control group (NC group). We first detected the migration of HSCs. HSC migration was significantly increased in the model group ( $<<0.05$, vs. the control group), while with FA treatment, HSC migration was significantly decreased ( $\mathrm{p}<0.05 \mathrm{vs}$. the model group), and $1 \mathrm{mg} / \mathrm{mL}$ FA showed an especially good inhibitory effect (Figure 6a-b). 
Western blot analysis confirmed that FA could promote BMSC-mediated inhibition of HSC activation, as indicated by assessment of HSC activation markers, including a-smooth muscle actin (a-SMA) and collagen type 1 a 1 chain (COL1-A1). The protein levels of a-SMA and COL1-A1 in the model group were significantly increased ( $\mathrm{p}<0.05$ vs. the NC group). However, the expression of a-SMA and COL1-A1 was significantly decreased after FA treatment, and 1 $\mathrm{mg} / \mathrm{mL}$ FA showed better effects ( $\mathrm{p}<0.05 \mathrm{vs}$. the model group). We also detected a-SMA and COL1-A1 in extracellular medium by elisa kits and found that the content of them was decreased (Figure 6c-d). After confirming the effect of FA on HSC inactivation, we further assessed the direct pathway between BMSCs and HSCs. The cytoskeleton changed when HSCs were activated. We chose the RhoA/ROCK/SRF and RhoA/ROCK/LIMK1 pathways. The protein levels of RhoA, ROCK, SRF, LIMK1 were significantly increased in the model group compared to the NC group ( $<<0.05)$, and with $1 \mathrm{mg} / \mathrm{mL} F A$ treatment, the expression of RhoA, ROCK, SRF, LIMK1 was significantly decreased. The other two dose groups showed no differences. The content of COL1-A1 and a-SMA in the ECM also decreased with $1 \mathrm{mg} / \mathrm{mL}$ FA treatment (Figure 6f-g). The mRNA levels showed the same trends (Figure 6e).

\section{BMSCs regulate the activation of HSCs by inhibiting cytoskeletal rearrangement}

To further prove that the cytoskeleton pathway acts as the direct interaction between BMSCs and HSCs that allows BMSCs to inhibit HSC activation, we added a cytoskeleton inhibitor (cytochalasin D) or cytoskeleton agonist (angiotensin II) when BMSCs were co-cultured with activated HSCs. Compared to the model group, the agonist group showed significantly increased protein expression of COL1-A1, a-SMA, RhoA, ROCK, SRF, and LIMK1 (p<0.05), while the expression levels of these proteins were significantly decreased in the inhibitor group $(p<0.05)$. The content of COL1-A1 and a-SMA in the ECM was also decreased with cytochalasin D treatment (Figure 7a-b). The content of COL1-A1 and a-SMA in the ECM also decreased with $1 \mathrm{mg} / \mathrm{mL}$ FA treatment (Figure 7d-e). The RNA levels of COL1-A1, a-SMA, RhoA, ROCK, SRF, and LIMK1 were reduced in the cytochalasin D groups (Figure 7c). These results indicate that FA promotes the ability of BMSCs to influence HSC activation. The cytoskeleton-related RhoA/ROCK pathway acts as the direct interaction between BMSCs and HSCs.

\section{miR-19b-3p derived from BMSCs inactivates HSCs by suppressing the expression of its target TGF- $\beta$ R2}

miRNAs are found in BMSCs, and miRNAs transferred into target cells can impact cell behaviors by regulating the expression of target genes. Because miRNAs derived from BMSCs influenced HSC activation, we assessed which miRNAs contained in BMSCs regulated HSC activation. In previous studies, 20 miRNAs were found to be relatively highly expressed in BMSCs. Among them, miR-19b was found to be potentially linked to fibrosis. Bioinformatic analysis using TargetScan and mirwalk predicted TGF- $\beta R 2$, a receptor for TGF- $\beta 1$, as a putative target of miR-19b-3p, and a luciferase reporter assay revealed that miR$19 \mathrm{~b}-3 \mathrm{p}$ directly bound to the $3^{\prime}$ untranslated region (UTR) of the TGF- $\beta$ R2 mRNA transcript (Figure 8a-b).

To examine whether miR-19b-3p impacted HSC activation, HSCs were transfected with a miR-19b-3p mimic, meaningless fragment (negative control). Additionally, we chose inactivated HSCs and activated HSCs as the normal and model controls, respectively. Although miR-19b-3p was rarely expressed in activated HSCs, its expression was greatly elevated in miR-19b-3p-transfected HSCs (Figure 8c). In addition, the profibrotic markers a-SMA, COL1-A1, RhoA, and ROCK1 were downregulated in HSCs transfected with the miR-19b-3p mimics compared with those in the model and inhibitor groups ( $\mathrm{p}<0.05$ ) (Figure 8de). The content of a-SMA and COL1-A1 in the ECM was also showed decreased trend in the mimic group (Figure 8f-g). Western blot assays confirmed the RNA data, showing reductions in TGF- $\beta$ R2 and profibrotic marker expression in HSCs transfected with miR-19b-3p. These results suggest that miR-19b-3p inhibits HSC activation by directly targeting TF- $\beta$ R2.

We also transfected the miR-19b-3p mimic, miR-19b-3p inhibitor or negative control with the fluorescent Cy3 gene into activated HSCs. With confocal scanning microscopy, we observed red fluorescence in the miR-19b-3p mimic and negative control groups. Compared to the model group, the group transfected with the miR-19b-3p mimics showed F-Actin mainly distributed in peripheral cells, and a few fine stress fibres were found in these cells. The cytoskeleton of cells in the negative control groups was similar to that of cells in the model group (Figure 8h).

\section{FA induces BMSCs to release more miR-19b-3p to HSCs and inhibit the activation of HSCs}

To determine whether BMSCs transfer miR-19b-3p to HSCs, we transfected the miR-19b-3p mimic or miR-19b-3p inhibitor with the fluorescent Cy3 gene into BMSCs and co-cultured the transfected cells with activated HSCs. With confocal scanning microscopy, we observed red fluorescence in the miR-19b-3p mimic group, and F-Actin was mainly distributed in peripheral cells. A few fine stress fibres were found in these cells compared to those in the model group. In addition, in the miR-19b-3p inhibitor group, the cell shape was similar to that in the model group, and F-Actin in activated HSCs was remodelled to form a large number of thick stress fibres across the whole cell. While with FA treated, we found more red fluorescence in the miR-19b-3p mimic groups (Figure 9a).

To assess protein levels, the BMSC/HSC co-culture system was divided into 8 groups: the normal, model, mimics, inhibitor, mimics+FA, inhibitor+FA, and negative groups. We detected a-SMA, COL1-A1, RhoA, ROCK1 SRF, and LIMK1 and found that their expression was significantly downregulated in the mimic, mimic+FA, and inhibitor+FA groups compared to the model group. The content of a-SMA and COL1-A1 in the ECM was also decreased in these 3 groups (Figure 9b-c). The content of a-SMA and COL1-A1 in the ECM was also showed decreased trend in the mimic\&FA group (Figure 9d-e). The mRNA levels also showed the same trends (Figure 9f).

These results demonstrate that miR-19b-3p plays a critical role in mediating the effect of BMSCs on liver fibrosis. Taken together, these results suggest that FA combined with BMSC treatment can induce greater release of miR-19b-3p, which inhibits the activation of HSCs by suppressing the RhoA/ROCK1/SRF and RhoA/ROCK1/LIMK1 pathways, contributing to alleviating liver fibrosis. 


\section{Discussion}

In this study, we identified a new mechanism by which FA and BMSCs treat liver fibrosis. We used BMSCs, FA or combination therapy to treat rats with hepatic fibrosis. By evaluation of AST, ALT, HE and Masson staining results, we observed that liver fibrosis was decreased to different degrees and that the combination therapy showed better effects than FA or BMSC monotherapy. In addition, we detected the protein expression of COL1-A1, a-SMA, ROCK1 and RhoA, and the results for the combination therapy also showed better effects. Therefore, these results suggest that FA can promote the ability of BMSCs to improve liver fibrosis by targeting the RhoA/ROCK pathway.

The RhoA/ROCK signaling pathway is ubiquitously related to intracellular cytosis, colonization, migration, apoptosis, gene expression and other behaviors ${ }^{[12,}$

13]. The RhoA/ROCK pathway also affects the formation of fibrosis. It promotes collagen and ECM production in fibroblasts and increases the transformation of fibroblasts into myofibroblasts expressing a-SMA and other activated factors ${ }^{[14,15]}$.

To verify the mechanism by which FA promotes BMSCs, we established a BMSC/HSC-T6 co-culture system. Activated HSCs were co-cultured with an equal amount of BMSCs. We observed the cellular structure of HSC-T6 cells and found that with FA treatment, the deformed cell structure was restored, F-Actin was mainly distributed in peripheral cells, and a few fine stress fibres were found in these cells. In addition to assessing cellular structure, we detected the migration of HSCs. The number of migration cells was significantly decreased with FA and BMSC treatment. The protein expression of RhoA, ROCK, SRF, LIMK1, COL1-A1 and a-SMA was significantly decreased. These results all proved that FA promoted the ability of BMSCs to decrease HSC migration, meanwhile, inhibit activation.

The RhoA/ROCK pathway is related to both fibrosis development and cytoskeletal rearrangement. RhoA/ROCK/SRF directly influences collagen and a-SMA production, while RhoA/ROCK/LIMK1 influences cytoskeletal rearrangement, indirectly affecting HSC migration. Activated HSCs migrate to sites of damage and release collagen. Therefore, inhibiting cytoskeletal rearrangement is also an effective strategy for reducing fibrosis ${ }^{[16-18]}$.

To verify the mechanism by which BMSCs inhibit HSC activation, we established a BMSC/HSC co-culture system with angiotensin II and cytochalasin D used as an agonist and inhibitor, respectively. The decreased protein expression of RhoA, ROCK, SRF, LIMK1, COL1-A1 and a-SMA also indicated that BMSCs could reduce HSC activation by inhibiting cytoskeletal rearrangement.

In a recent study, BMSCs were found to release miRNAs, targeting specific proteins to reduce fibrosis ${ }^{[19,20]}$. The results for the BMSC genomic sequence showed that BMSCs contain a variety of miRNAs ${ }^{[21,22]}$. Among them, miR-19b-3p was shown to be related to inhibiting fibrosis ${ }^{[23-26]}$. We used the TargetScan website to predict the target gene of miR-19b-3p and found TGF- $\beta R 2$ to be a putative target. The luciferase reporter assay revealed that miR-19b-3p directly bound to TGF- $\beta$ R2 in HSCs.

To identify whether miR-19b-3p can inhibit HSC activation, we transfected miR-19b-3p mimics or inhibitors with the Cy3 gene into HSCs and BMSCs. We observed the cytoskeleton of HSCs transfected with the miR-19b-3p mimics or inhibitors. With miR-19b-3p mimic transfection, the cytoskeleton of activated HSCs was not changed considerably, while with miR-19b-3p inhibitor transfection, the cytoskeleton of activated HSCs was still rearranged. The decreased expression of RhOA, ROCK, SRF, LIMK1, COL1-A1 and a-SMA showed that miR-19b-3p could affect HSC activation.

We also observed that BMSCs transfected with the mimics released more miR-19b-3p to HSCs and that the cytoskeleton was similar to that of inactivated HSCs; BMSCs transfected with the inhibitors released less miR-19b-3p to HSCs, and the cytoskeleton was similar to that of activated HSCs. The protein expression of RhoA, ROCK, SRF, LIMK1, COL1-A1 and a-SMA showed that BMSCs could release miR-19b-3p to inhibit HSC activation, and BMSCs combined with FA treatment showed better HSC inhibition. These results support the notion that combining BMSCs with FA could be a promising therapeutic strategy for the treatment of liver disease.

Bone marrow has been the most widely used source of MSCs. However, the use of these cells in clinical applications has been limited due to the instability of BMSCs $^{[27-29]}$. FA is a derivative of cinnamic acid and has therapeutic activity against a variety of diseases. It is also an effective ingredient in some traditional Chinese medicines, such as Angelica Sinensis. In addition, FA may protect against fibrotic deterioration in rats with hepatic fibrosis ${ }^{[30]}$. Therefore, we chose FA combined with BMSCs to treat hepatic fibrosis in rats, and found that their combination showed better effects on treatment.

\section{Conclusion}

Collectively, we demonstrated that FA promoted the ability of BMSCs to suppress HSC activation and liver fibrosis. MiR-19b-3p derived from BMSCs inactivated HSCs by suppressing the RhoA/ROCK/SRF and RhoA/ROCK/LIMK1 signaling pathways. Therefore, our findings suggest that BMSCs combined with a traditional Chinese medicine have great potential as an effective antifibrotic therapeutic agent for treating chronic liver disease.

\section{Abbreviations}

SD rat: Sprague Dawley rat; ANOVA: Analysis of variance; DAPI: Diamidinophenylindole; DMEM: Dulbecco's modifed Eagle's medium; FA: Ferulic acid; FBS: Fetal bovine serum; PBS: Phosphate bufer saline; BMSCs: Bone marrow mesenchymal stem cells H\&E: Hematoxylin and eosin; ROCK1: Rho-associated kinase 1; RhoA: Ras homolog family member A; SRF: Serum response factor; LIMK1: LIM domain kinase 1; COL1-A1: Collagen type I alpha 1 chain; a-SMA: Alphasmooth muscle actin; TGF- $\beta 1 \otimes$ Transforming growth factor beta 1;TGF- $\beta$ R2:Transforming growth factor beta receptor 2; ECM: Extracellular matrix; HSCs: Hepatic stellate cells.

\section{Declarations}




\section{Contribution}

Rui Zhang as principal author was responsible processing experiments, collecting and analyzing data, finally independent wrote this manuscript. Wenlan Liu and Jiabo Wang performed design of study and reviewed manuscript, and acted as corresponding author. Wenhang Li, Xiandan Jiang and Xinyi Cui contributed to complete experiments. Hongjie You and Zuoqing Tang contributed to data interpretation.

\section{Supplementary Information}

Not applicable.

\section{Acknowledgements}

Not applicable.

\section{Fundings}

This work supported by grants from National Natural Science Foundation of China (to Wenlan Liu.) (Grant No. 81573879), Natural Science Foundation of Beijing (to Wenlan Liu.) (Grant No.7182021).

\section{Availability of data and materials}

All data generated and/or analyzed in this study are available from corresponding authors upon reasonable request.

\section{Ethics approval and consent to participate}

All experiments involving animals were performed in accordance with guidelines approved by the Institutional Animal Care and Use Committee of Capital Medical University, P.R. China.

\section{Consent for publication}

Not applicable.

\section{Competing interests}

The authors declare that they have no competing interests.

\section{References}

1. Brenner RBDA. Liver fibrosis. J Clin Investig. 2005;115:209-18. DOI:10.1172/JCI200524282.

2. Pinzani M. KR. Erratum to "liver fibrosis: From the bench to clinical targets". Digestive Liver Disease. 2004;36:562-3. D0I:10.1016/j.dld.2004.06.001.

3. Virarkar M, Morani AC, Taggart MW, Bhosale P. Liver fibrosis assessment. Semin Ultrasound CT MR. 2021;42:381-9. DOI:10.1053/j.sult.2021.03.003.

4. Alfaifi M, Eom YW, Newsome PN, Baik SK. Mesenchymal stromal cell therapy for liver diseases. J Hepatol. 2018;68:1272-85. DOI:10.1016/j.jhep.2018.01.030.

5. Li R, Luo X, Zhu Y, Zhao L, Li L, Peng Q, Ma M, Gao Y. Atm signals to ampk to promote autophagy and positively regulate DNA damage in response to cadmium-induced ros in mouse spermatocytes. Environ Pollut. 2017;231:1560-8. DOI:10.1016/j.envpol.2017.09.044.

6. Cetin Z, Saygili El, Gorgisen G, Sokullu E. Preclinical experimental applications of mirna loaded bmsc extracellular vesicles. Stem Cell Rev Rep. 2021;17:471-501. DOI:10.1007/s12015-020-10082-x.

7. Feng Y, Huang W, Wani M, Yu X, Ashraf M. Ischemic preconditioning potentiates the protective effect of stem cells through secretion of exosomes by targeting mecp2 via mir-22. PLoS One. 2014;9:e88685. DOI:10.1371/journal.pone.0088685.

8. Hyun J, Wang S, Kim J, Kim GJ, Jung Y. Microrna125b-mediated hedgehog signaling influences liver regeneration by chorionic plate-derived mesenchymal stem cells. Sci Rep. 2015;5:14135. DOI:10.1038/srep14135.

9. Jayamani J, Naisini A, Madhan B, Shanmugam G. Ferulic acid, a natural phenolic compound, as a potential inhibitor for collagen fibril formation and its propagation. Int J Biol Macromol. 2018;113:277-84. DOI:10.1016/j.jjbiomac.2018.01.225.

10. Li D, Rui YX, Guo SD, Luan F, Liu R, Zeng N. Ferulic acid: A review of its pharmacology, pharmacokinetics and derivatives. Life Sci. 2021;284:119921. DOI:10.1016/j.Ifs.2021.119921.

11. Ali SA, Saifı MA, Pulivendala G, Godugu C, Talla V. Ferulic acid ameliorates the progression of pulmonary fibrosis via inhibition of tgf-beta/smad signalling. Food Chem Toxicol. 2021;149:111980. DOI:10.1016/j.fct.2021.111980.

Page 9/19 
12. Xu A, Li Y, Zhao W, Hou F, Li X, Sun L, Chen W, Yang A, Wu S, Zhang B, Yao J, Wang H, Huang J. Php14 regulates hepatic stellate cells migration in liver fibrosis via mediating tgf-beta1 signaling to pi3kgamma/akt/rac1 pathway. J Mol Med (Berl). 2018;96:119-33. D0I:10.1007/s00109-017-1605-6.

13. Yaqoob U, Luo F, Greuter T, Jalan Sakrikar N, Sehrawat TS, Lu J, Hu X, Gao J, Kostallari E, Chen J, Arab JP, Martin-Mateos R, Cao S, Shah VH. Gipcregulated igfbp-3 promotes hsc migration in vitro and portal hypertension in vivo through a beta1-integrin pathway. Cell Mol Gastroenterol Hepatol. 2020;10:545-59. DOI:10.1016/j.jcmgh.2020.05.005.

14. Zhenhan Deng YJ, Haifeng Liu M, He Y, Yang WX, Li Y. Rhoa/rock pathway: Implication in osteoarthritis and therapeutic targets. Am J Transl Res. 2019;11:5324-31.

15. Sloniecka M, Danielson P. Substance $\mathrm{p}$ induces fibrotic changes through activation of the rhoa/rock pathway in an in vitro human corneal fibrosis model. J Mol Med (Berl). 2019;97:1477-89. DOI:10.1007/s00109-019-01827-4.

16. Ni J, Dong Z, Han W, Kondrikov D, Su Y. The role of rhoa and cytoskeleton in myofibroblast transformation in hyperoxic lung fibrosis. Free Radic Biol Med. 2013;61:26-39. DOI:10.1016/j.freeradbiomed.2013.03.012.

17. Lok AS, McMahon BJ, Practice Guidelines Committee AAftSoLD. Chronic hepatitis b Hepatology. 2001;34:1225-41. DOI:10.1053/jhep.2001.29401.

18. Ying Wu ZL, Wang S, Xiu A, Zhang C. Carvedilol inhibits angiotensin ii-induced proliferation and contraction in hepatic stellate cells through the rhoa/rhokinase pathway. Biomed Res Int. 2019;6:1-15.

19. Chen L, Lu FB, Chen DZ, Wu JL, Hu ED, Xu LM, Zheng MH, Li H, Huang Y, Jin XY, Gong YW, Lin Z, Wang XD, Chen YP. Bmscs-derived mir-223-containing exosomes contribute to liver protection in experimental autoimmune hepatitis. Mol Immunol. 2018;93:38-46. DOI:10.1016/j.molimm.2017.11.008.

20. BI Hui-yang GJ-I. Research progress of mirna related to bone marrow mesenchymal stem cells. World Latest Medicine Information (Electronic Version). 2019;19:68-9. DOI:10.19613/j.cnki.1671-3141.2019.52.035.

21. Zhao XK, Yu L, Cheng ML, Che P, Lu YY, Zhang Q, Mu M, Li H, Zhu LL, Zhu JJ, Hu M, Li P, Liang YD, Luo XH, Cheng YJ, Xu ZX, Ding Q. Focal adhesion kinase regulates hepatic stellate cell activation and liver fibrosis. Sci Rep. 2017;7:4032. DOI:10.1038/s41598-017-04317-0.

22. Lindoso RS, Collino F, Bruno S, Araujo DS, Sant'Anna JF, Tetta C, Provero P, Quesenberry PJ, Vieyra A, Einicker-Lamas M, Camussi G. Extracellular vesicles released from mesenchymal stromal cells modulate mirna in renal tubular cells and inhibit atp depletion injury. Stem Cells Dev. 2014;23:1809-19. DOI:10.1089/scd.2013.0618.

23. Duan L, Duan D, Wei W, Sun Z, Xu H, Guo L, Wu X. Mir-19b-3p attenuates il-1beta induced extracellular matrix degradation and inflammatory injury in chondrocytes by targeting grk6. Mol Cell Biochem. 2019;459:205-14. DOI:10.1007/s11010-019-03563-2.

24. Wang Y, Wang L, Guo J, Zuo S, Wang Z, Hua S. Mypt1, regulated by mir-19b-3p inhibits the progression of non-small cell lung cancer via inhibiting the activation of wnt/beta-catenin signaling. Life Sci. 2021;278:119573. DOI:10.1016/j.Ifs.2021.119573.

25. Chen W, Zhou ZQ, Ren YQ, Zhang L, Sun LN, Man YL, Wang ZK. Effects of long non-coding rna linc00667 on renal tubular epithelial cell proliferation, apoptosis and renal fibrosis via the mir-19b-3p/linc00667/ctgf signaling pathway in chronic renal failure. Cell Signal. 2019;54:102-14. DOI:10.1016/j.cellsig.2018.10.016.

26. Zou M, Wang F, Gao R, Wu J, Ou Y, Chen X, Wang T, Zhou X, Zhu W, Li P, Qi LW, Jiang T, Wang W, Li C, Chen J, He Q, Chen Y. Autophagy inhibition of hsamir-19a-3p/19b-3p by targeting tgf-beta r ii during tgf-beta1-induced fibrogenesis in human cardiac fibroblasts. Sci Rep. 2016;6:24747. DOI:10.1038/srep24747.

27. Yang X, Chen Z, Chen C, Han C, Zhou Y, Li X, Tian H, Cheng X, Zhang K, Qin A, Zhou T, Zhao J. Bleomycin induces fibrotic transformation of bone marrow stromal cells to treat height loss of intervertebral disc through the tgfbetar1/smad2/3 pathway. Stem Cell Res Ther. 2021;12:34. DOI:10.1186/s13287-02002093-9.

28. Alsulami M, Abdel-Gaber R. Cell therapy as a new approach on hepatic fibrosis of murine model of schistosoma mansoni-infection. Acta Parasitol. 2021;66:136-45. DOI:10.1007/s11686-020-00267-2.

29. Muslimah Alsulami RAG. Exosomal transfer of bone marrow mesenchymal stem. Acta Parasitologica. 2021;66:136-45.

30. Mu M, Zuo S, Wu RM, Deng KS, Lu S, Zhu JJ, Zou GL, Yang J, Cheng ML, Zhao XK. Ferulic acid attenuates liver fibrosis and hepatic stellate cell activation via inhibition of tgf-beta/smad signaling pathway. Drug Des Devel Ther. 2018;12:4107-15. DOI:10.2147/DDDT.S186726.

\section{Figures}


(a)
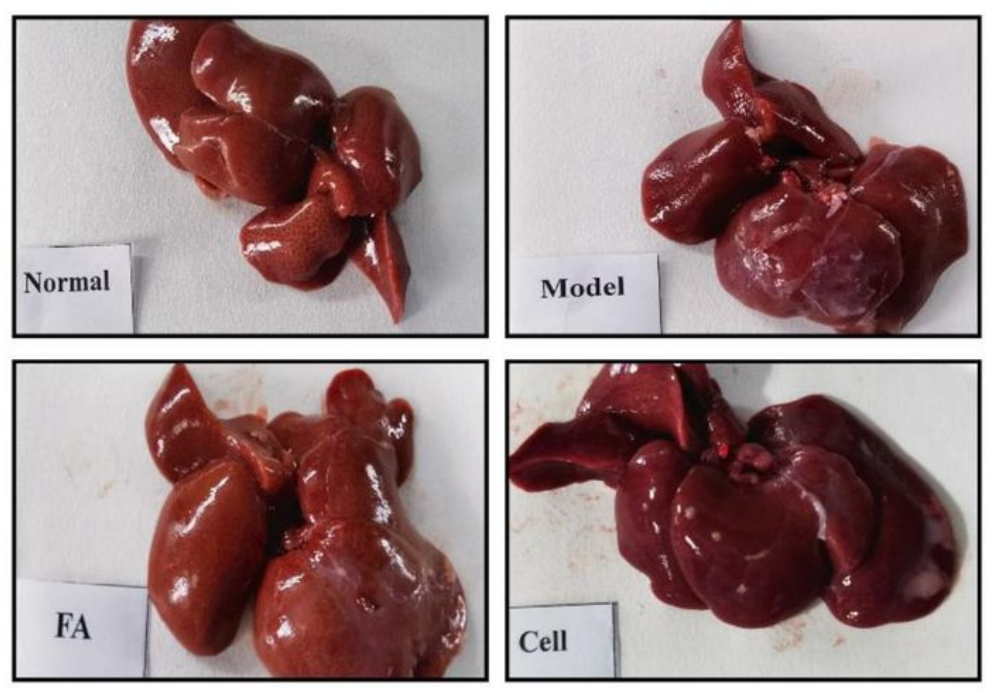

(c)

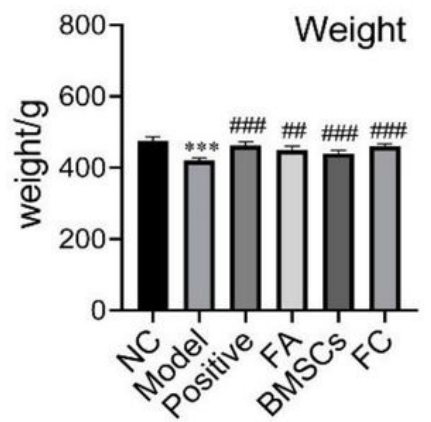

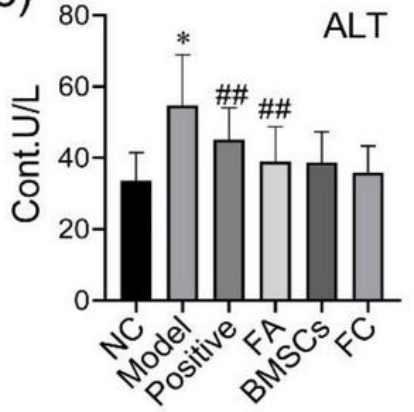
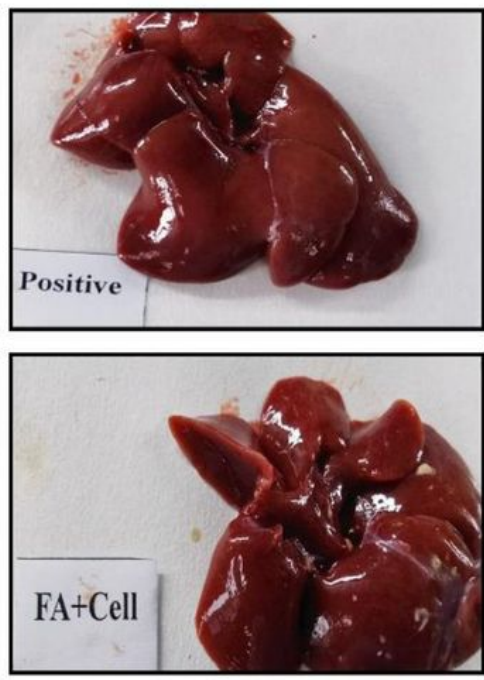

(d)

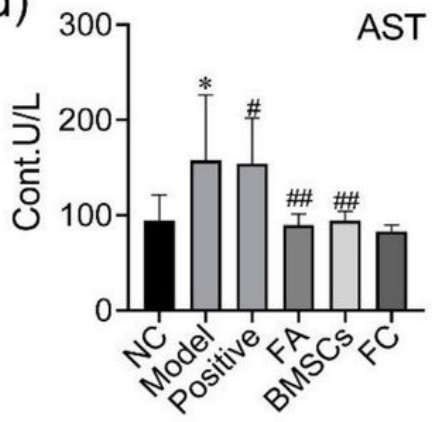

Figure 1

Administration of BMSCs combined with FA ameliorates $\mathrm{CCl}_{4}$-induced liver injury in rats. (a) The liver appearance of each group (normal, model, positive, BMSC, FA and FC groups). (b) Comparison of body weight among the groups (two-way ANOVA; ${ }^{\star} p<0.05,{ }^{* \star} p<0.01,{ }^{\star \star \star} p<0.001$ versus normal; \#p<0.05, \#\#p<0.01, \#\#\#p<0.001 versus model). (c)\&(d) The serum levels of AST and ALT in each group $(n=6)$. Data are presented as the mean \pm SEM (two-way ANOVA; * $p<0.05$ versus normal; \#p<0.05, \#\#p<0.01 versus model). 
(a)
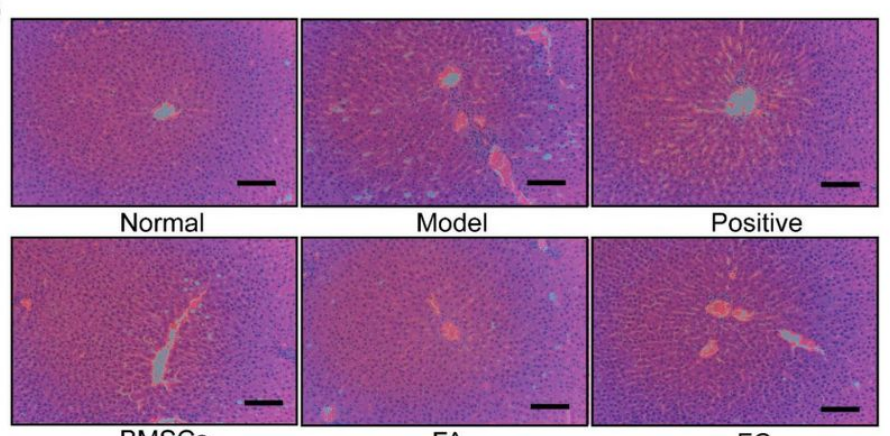

(b)
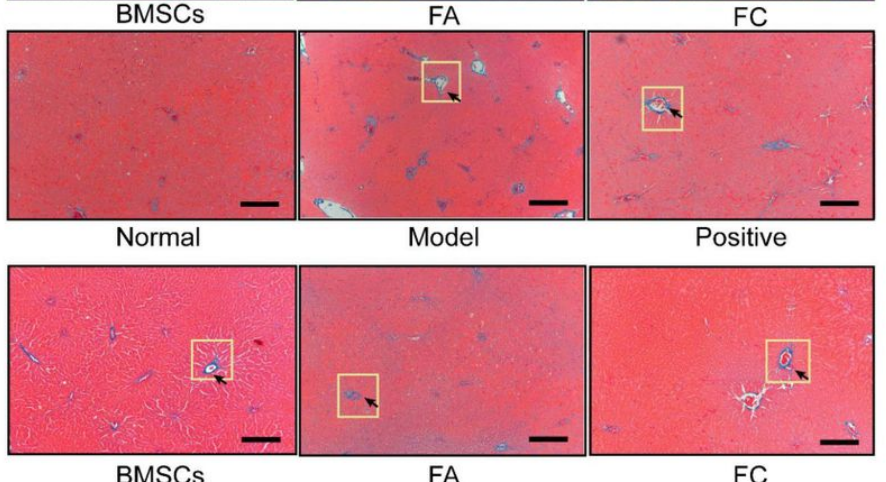

(c)

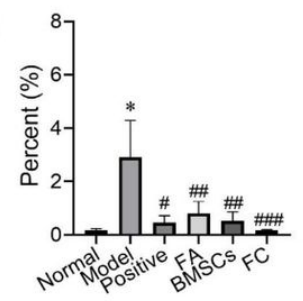

Figure 2

(a) Representative images of haematoxylin and eosin-stained liver sections from representative animals in the normal, model, positive, BMSC, FA and FC groups (original magnification, 10x; scale bars, $100 \mathrm{um}$ ). (b) Representative images of Sirius red-stained liver sections from representative animals in the normal, model, positive, BMSC, FA and BMSC\&FA groups (original magnification, 10x; scale bars, $100 \mathrm{um}$ ). The yellow squares mark the collagen. (c) The percentage of collagen area of each group $(n=6)$. Data are presented as the mean \pm SEM (two-way ANOVA; ${ }^{*}<0.05$ versus normal; \#p<0.05, \#\#p<0.01, \#\#\#p0.001 versus model.)

Tracking BMSCs in vivo 


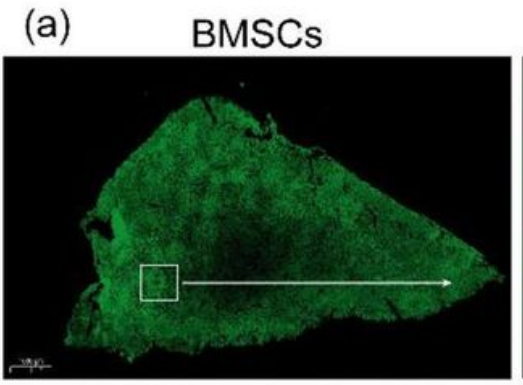

BMSCs\&Ferulic acid

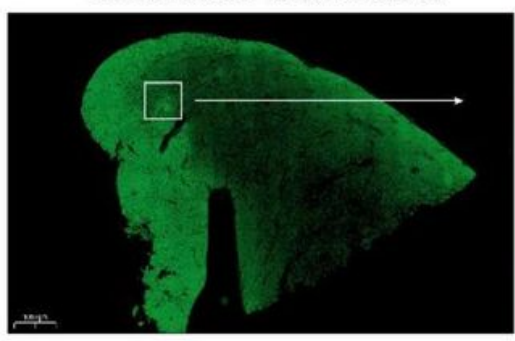

Magnification

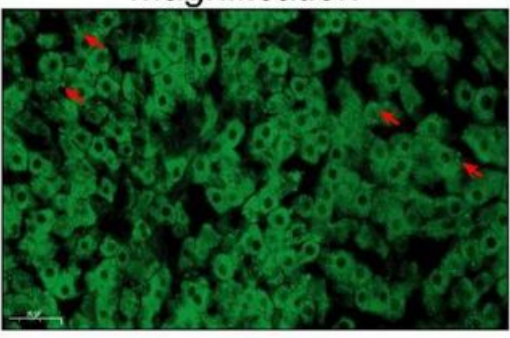

Magnification

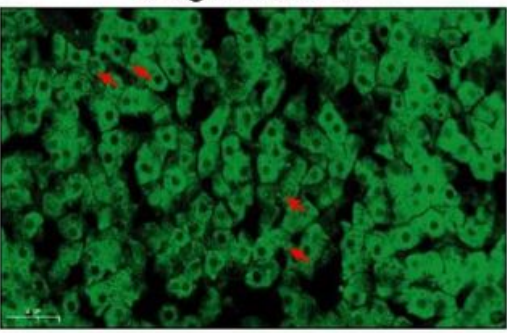

(b)

Light-sheet microscopy

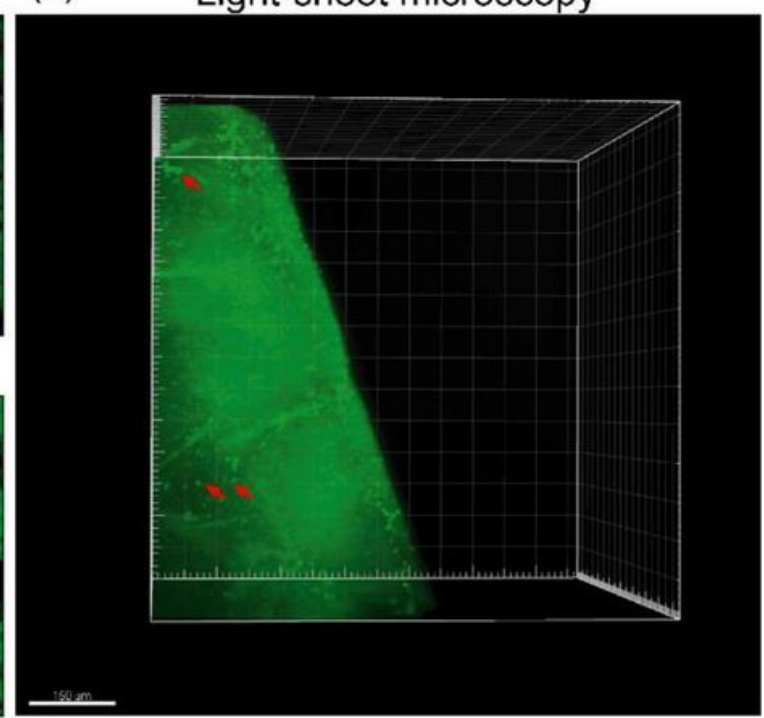

Figure 3

(a) Representative images of frozen liver sections from representative animals in the BMSC and BMSC+FA groups (original magnification, 10x; scale bars, 10 um and 50um). (b) Representative images of light-sheet liver sections from the BMSC+FA group (original magnification, 10x; scale bars, 150 um). The red arrow labeled cell location. 


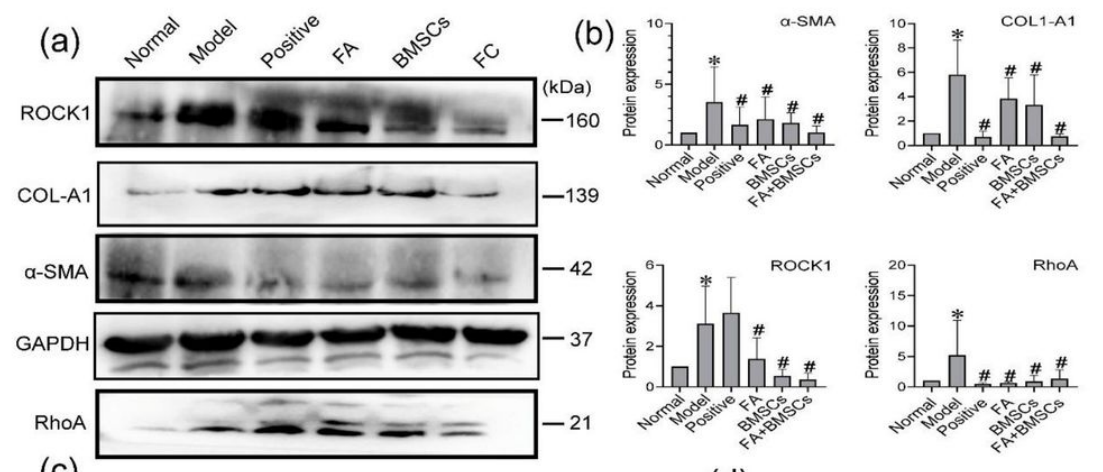

(c)
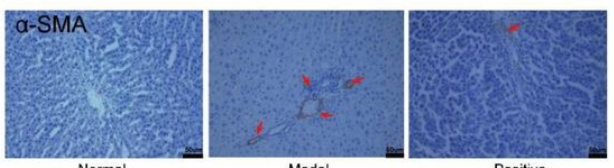

(d)

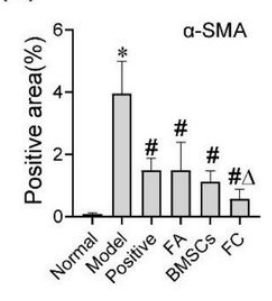

(e)
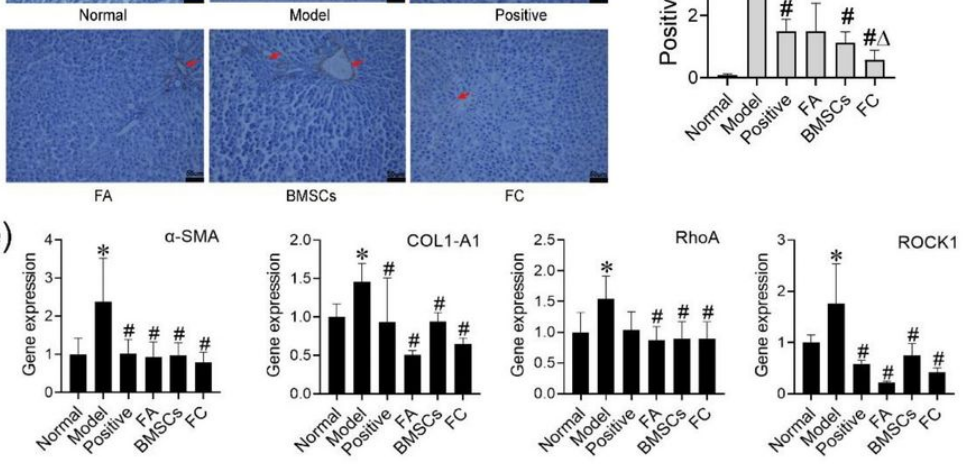

Figure 4

( $a$ and b) Westem blot and cumulative densitometric analyses of ROCK1 (160 kDa), COL1-A1 (139 kDa), a-SMA (42 kDa), GAPDH (37 kDa) and RhoA (21 kDa) in livers from each group (Normal, Model, Positive,FA,BMSCs,FC). Band densities were normalized to the expression level of GAPDH, which was used as an intemal control. Data are presented as the mean \pm SEM ( $n=3$ independent experiments, two-way ANOVA; $* p<0.05$ versus normal; $\# p<0.05$, versus model); (c and d) The protein expression levels of a-SMA in each group: (c) indicates the a-SMA protein; (d) indicates the protein expression trends; Data are presented as the mean \pm SEM ( $n=3$ independent experiments, two-way ANOVA; ${ }^{*}<<0.05$, versus the control; \# $p<0.05$, versus the model group; $\triangle p<0.05$, versus the $B M S C s$ group) ; (e) qRT-PCR analysis for a-SMA, COL1-A1, ROCK1, and RhOA in rats' liver. Data are presented as mean \pm SEM of experiments performed in triplicate ( $n=3$ independent experiments, two-way ANOVA; ${ }^{p} p<0.05$ versus normal; \#p<0.05 versus model). 

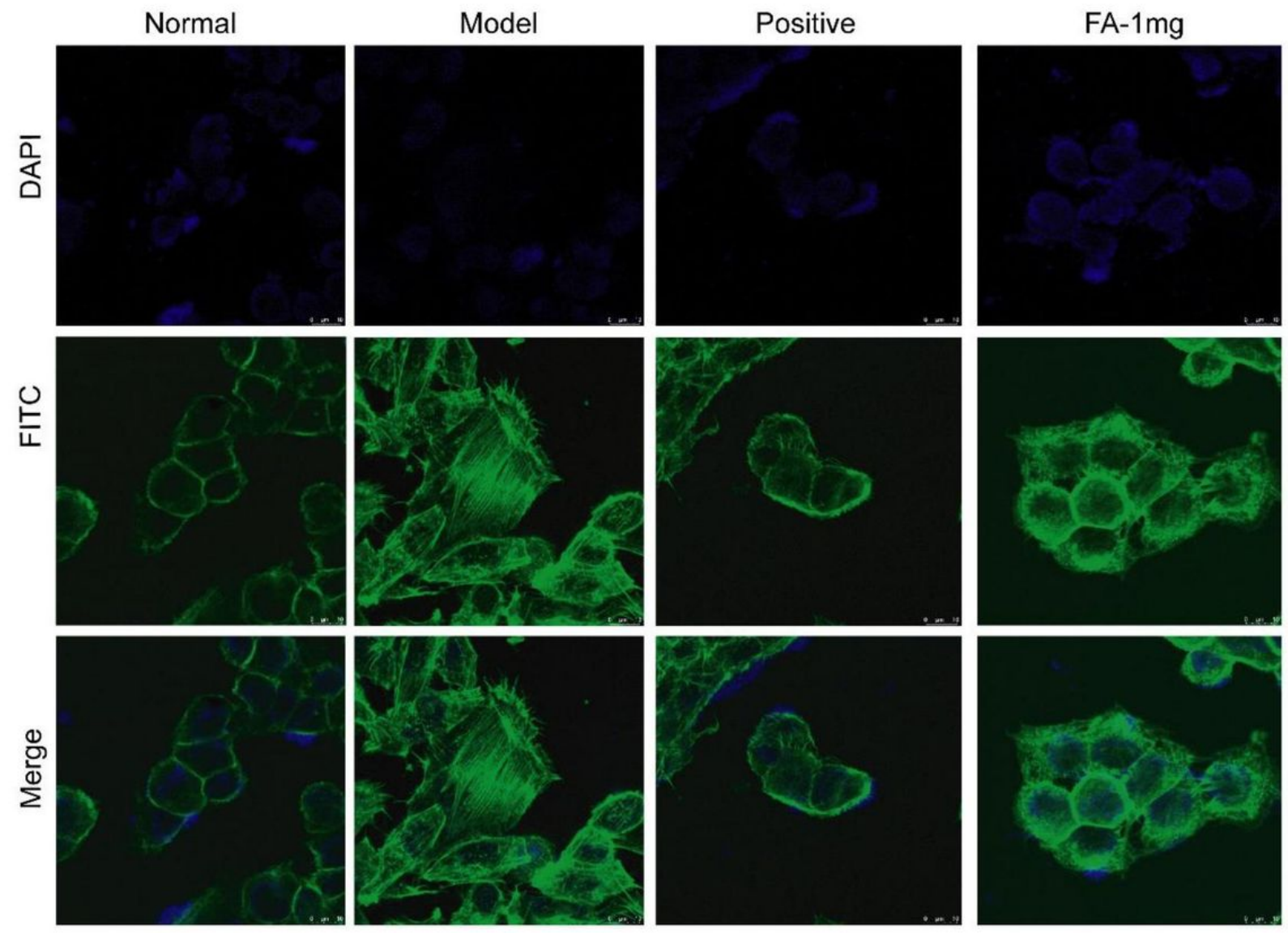

Figure 5

Representative images of the cytoskeleton (green) in HSCs treated with BMSCs or BMSC\&FA ( $1 \mathrm{mg} / \mathrm{mL}$ ). DAPI was used to stain cell nuclei (original magnification, 40x; scale bars: 10 um). 
(a)
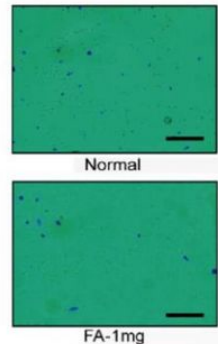

(c)

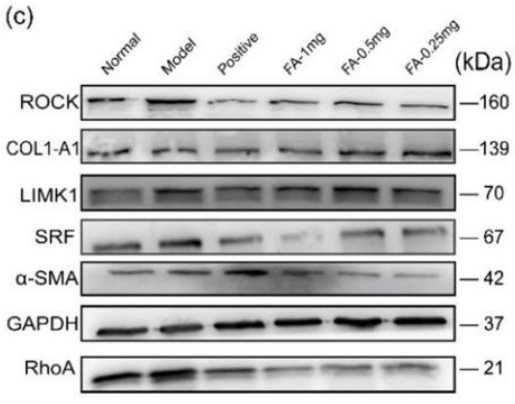

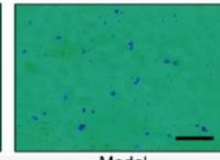

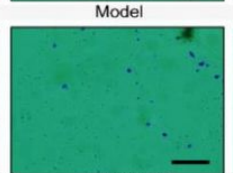

. (d)
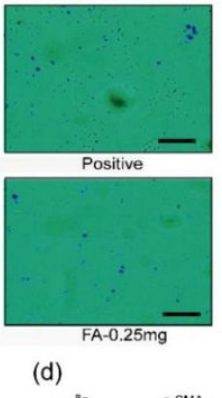

(b)

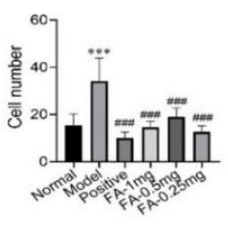

(e)
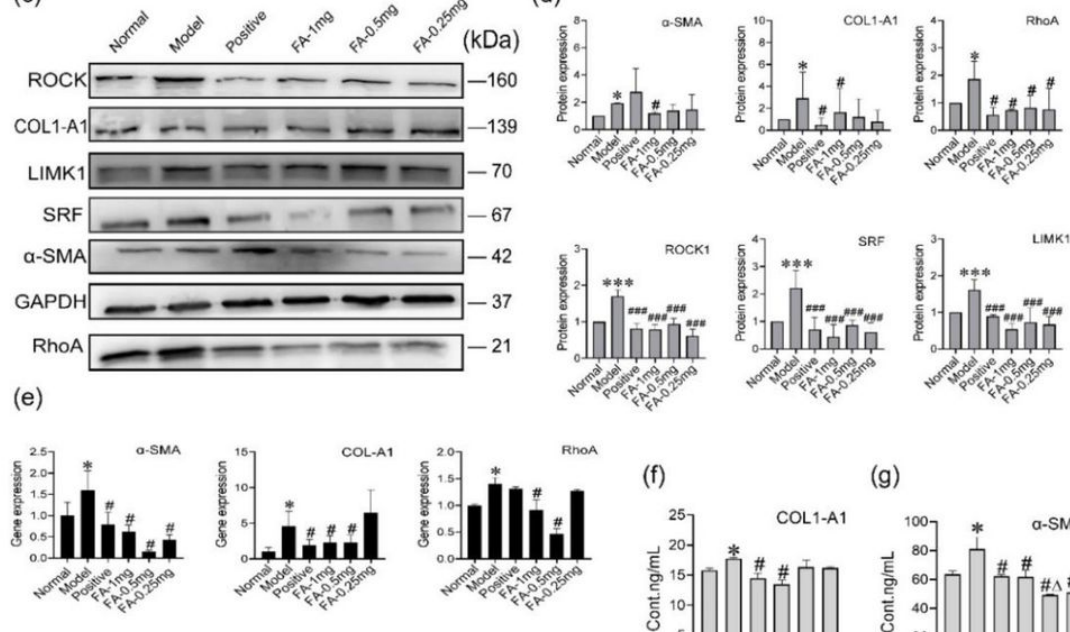

(f)

(g)
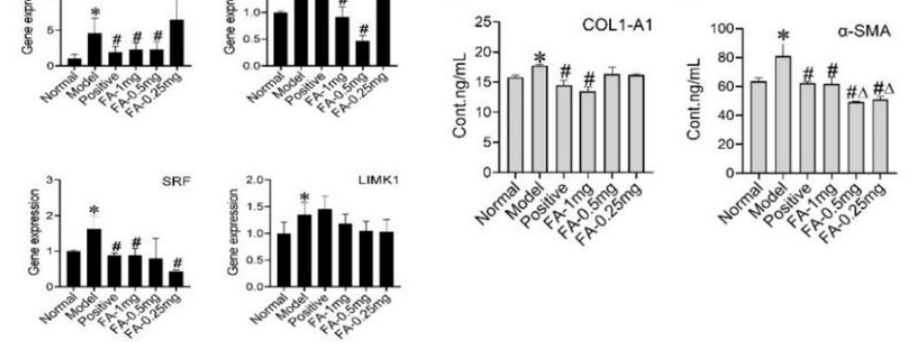

Figure 6

FA promotes the ability of BMSCs to inhibit the activation of HSCs. (a) Migration of HSCs (original magnification, 10x; scale bars, 100 um). (b) The number of migration HSCs. Data are presented as the mean \pm SEM ( $n=3$ independent experiments, two-way ANOVA; *** $<<0.001$ versus normal; \#\#\#p<0.001, versus model); (c-d) Western blot bands and cumulative densitometric analyses of each group(Normal, Model, Positive, FA-1mg, FA-0.5mg, FA-0.25mg). Data are presented as the mean \pm SEM ( $n=3$ independent experiments, two-way ANOVA; ${ }^{*}<<0.05$, ${ }^{\star * *} p<0.001$, versus normal; \#p<0.05, \#\#\#p<0.001, versus model); (e) qRT-PCR analysis for a-SMA, COL1-A1, ROCK1, RhOA, SRF, LIMK1 in HSCs. Data are presented as mean \pm SEM of experiments performed in triplicate ( $\mathrm{n}=3$ independent experiments, two-way ANOVA; ${ }^{*} p<0.05$ versus normal; \#p<0.05 versus model); ( $\left.-\mathrm{g}\right)$ The protein content of $a-S M A$ and COL1-A1 in extracellular medium. Data are presented as mean \pm SEM of experiments performed in triplicate ( $n=3$ independent experiments, two-way ANOVA; * $p<0.05$ versus normal; $\# p<0.05$ versus model; $\Delta \mathrm{p}<0.05$ versus $F A-1 \mathrm{mg}$.) 


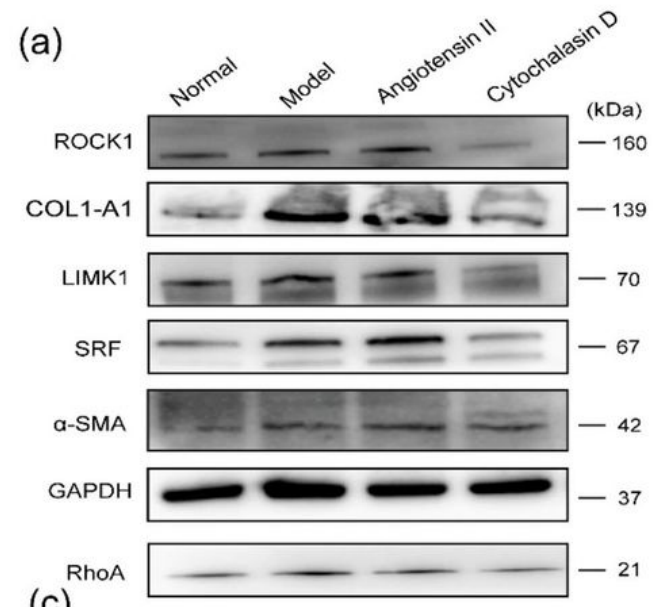

(b)
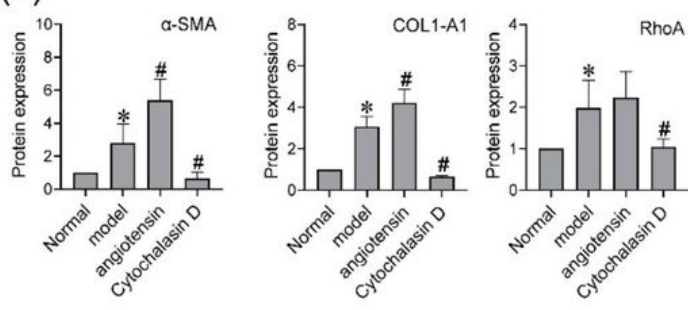

(c)
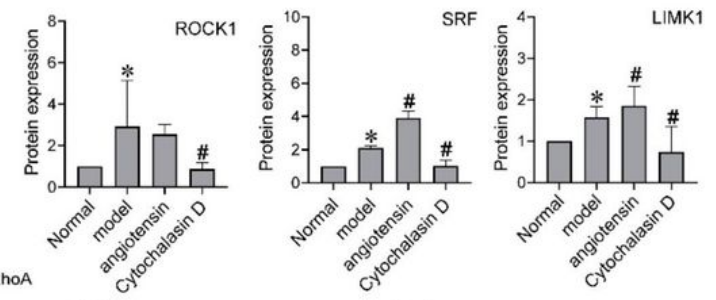
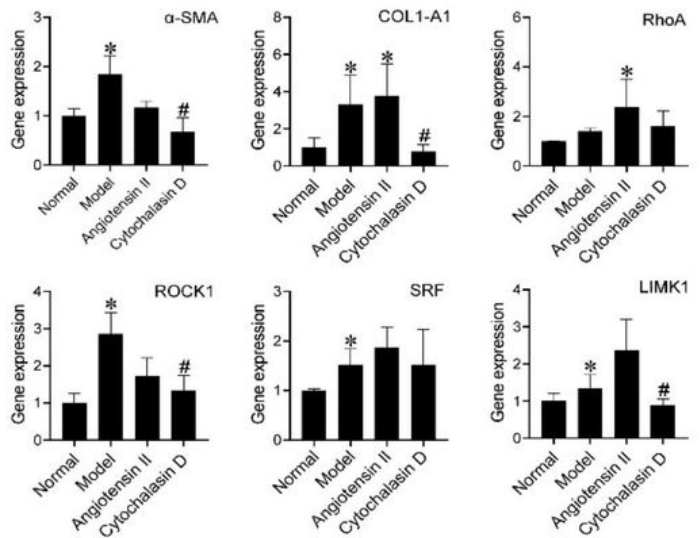

(d)

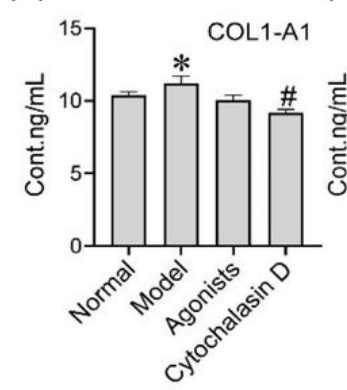

(e)

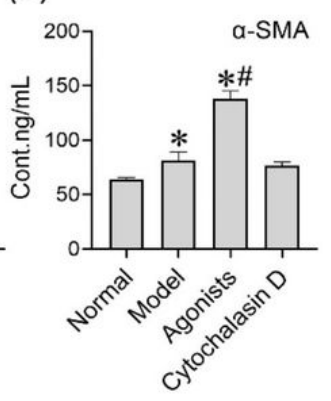

Figure 7

BMSCs regulate the activation of HSCs by inhibiting cytoskeletal rearrangement. (a-b) Western blot bands and cumulative densitometric analyses of each group (Normal, Model, Angiotensin II, Cytochalasin D). Data are presented as the mean \pm SEM ( $n=3$ independent experiments, one-way ANOVA; $* p<0.05$, versus normal; \#p<0.05, versus model); (c) qRT-PCR analysis for a-SMA, COL1-A1, ROCK1, RhoA, SRF, LIMK1 in HSCs. Data are presented as mean \pm SEM of experiments performed in triplicate ( $n=3$ independent experiments, one-way ANOVA; ${ }^{*}<0.05$ versus normal; \#p<0.05 versus model); ( $\mathrm{d}-\mathrm{e}$ ) The protein content of a-SMA and COL1-A1 in extracellular medium. Data are presented as mean \pm SEM of experiments performed in triplicate ( $n=3$ independent experiments, oneway ANOVA; * $p<0.05$ versus normal; \# $<<0.05$ versus model.). 


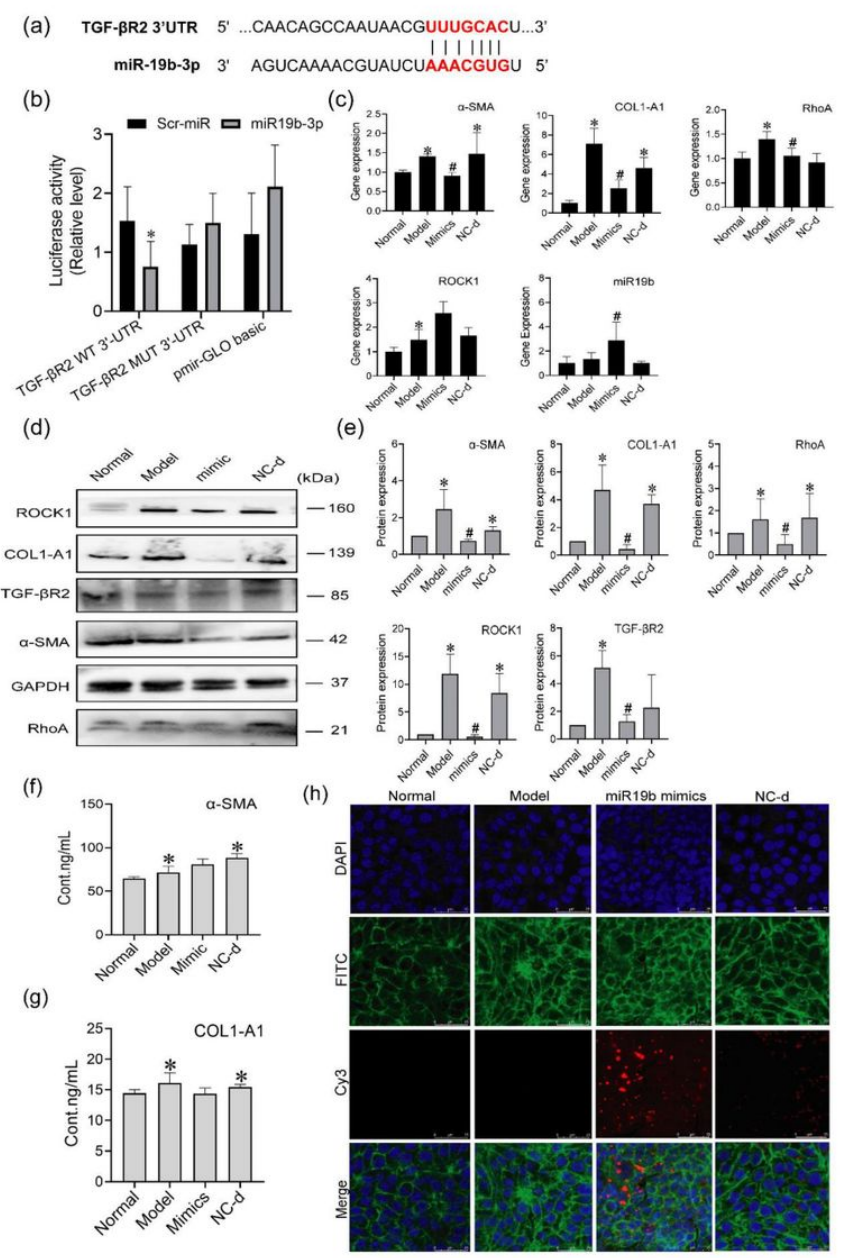

Figure 8

miR-19b-3p derived from BMSCs inactivates HSCs by suppressing the expression of its target TGF- $\beta$ R2. (a) The potential binding site (red fonts) of miR-19b$3 p$ was predicted in the $3^{\prime} U T R$ of TGF- $\beta R 2$ mRNA in rats. The dashed line represents complementary base pairs between miR-19b-3p and TGF- $\beta R 2$ mRNA; (b) A dual-luciferase assay was performed to verify binding interaction between miR-19b-3p and TGF- $\beta R 2$ mRNA. Cells co-transfected with pmir-GLO basic vector containing either wild-type (WT) or mutant (mut) target sites plus either the miR-19b-3p mimic or scrambled (Scr-) miR (control); (c) qRT-PCR analysis for aSMA, COL1-A1, ROCK1, RhoA in HSCs. Data are presented as mean \pm SEM of experiments performed in triplicate ( $n=3$ independent experiments, two-way ANOVA; * $p<0.05$ versus normal; \#p<0.05 versus model); (d-e) Western blot bands and cumulative densitometric analyses of each group (Normal, Model, mimic,NC-d). Data are presented as the mean \pm SEM ( $n=3$ independent experiments, two-way ANOVA; ${ }^{p}<0.05$, versus normal; \#p<0.05, versus model); $(f-g)$ The protein content of a-SMA and COL1-A1 in extracellular medium. Data are presented as mean \pm SEM of experiments performed in triplicate ( $n=3$ independent experiments, two-way ANOVA; ${ }^{*}$ < 0.05 versus normal; \#p<0.05 versus model); (h) Representative images of the cytoskeleton (green) in HSCs treated with miR19b-3p mimics or NC-d. DAPI was used to stain cell nuclei (original magnification, 40x; scale bars: 25 um). 


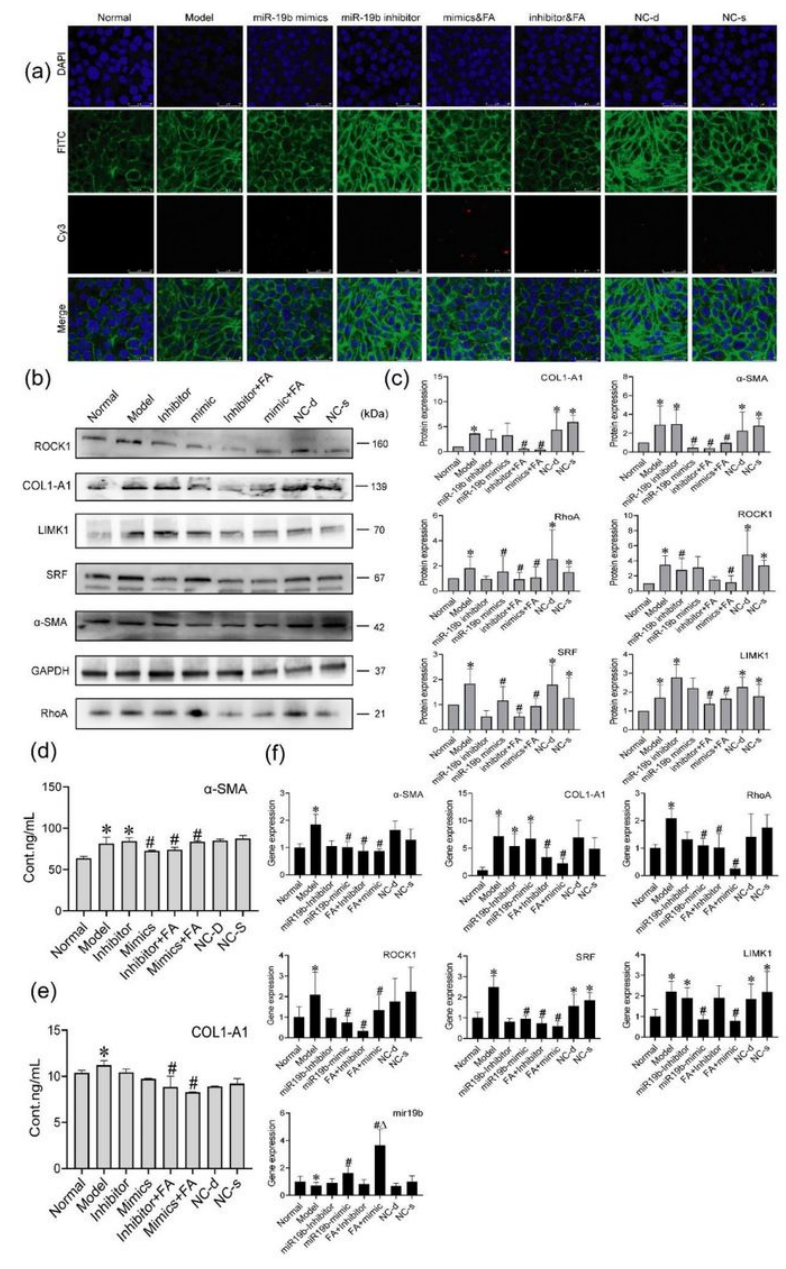

Figure 9

FA induces BMSCs to release more miR-19b-3p to HSCs and inhibit the activation of HSCs. (a) Representative images of the cytoskeleton (green) in HSCs treated with miR19b-3p mimics, inhibitor, or NC-d and NC-s. DAPI was used to stain cell nuclei (original magnification, 40x; scale bars: 25 um); (b-c) Western blot bands and cumulative densitometric analyses of each group (Normal, Model, inhibitor, mimic, inhibitor\&FA, mimic\&FA, NC-d, NC-s). Data are presented as the mean \pm SEM ( $n=3$ independent experiments, two-way ANOVA; * $p<0.05$, versus normal; \#p<0.05, versus model); (d-e) The protein content of $a-S M A$ and COL1-A1 in extracellular medium. Data are presented as mean \pm SEM of experiments performed in triplicate ( $n=3$ independent experiments, two-way ANOVA; ${ }^{*} p<0.05$ versus normal; \#p<0.05 versus model); (f) qRT-PCR analysis for a-SMA, COL1-A1, ROCK1, RhoA, SRF and LIMK1 in HSCs. Data are presented as mean \pm SEM of experiments performed in triplicate $\left(n=3\right.$ independent experiments, two-way ANOVA; ${ }^{*}<0.05$ versus normal; \#p<0.05 versus model; $\Delta p<0.05$ versus miR19b mimic).

\section{Supplementary Files}

This is a list of supplementary files associated with this preprint. Click to download.

- WBbands.rar 